\title{
MicroRNA-19a mediates neuroprotection through the PTEN/AKT pathway in SK-N-SH cells after oxygen-glucose deprivation/reoxygenation injury
}

\author{
Xiaochun Jin ${ }^{1, *}$, Hui Wang ${ }^{2, *}$, Shuzhou Yin ${ }^{1}$ and Youtao Zhang ${ }^{3}$ \\ ${ }^{1}$ Department of Anesthesiology, Suzhou Kowloon Hospital, Shanghai Jiaotong University School of Medicine, Suzhou, \\ Jiangsu, People's Republic of China \\ ${ }^{2}$ Department of Neurology, First Affiliated Hospital of Soochow University, Ping-hai Road, Suzhou, Jiangsu, 215006, People’s \\ Republic of China \\ ${ }^{3}$ Department of Clinical Laboratory, First Affiliated Hospital of Soochow University, Suzhou, Jiangsu, 215006, People’s \\ Republic of China
}

\begin{abstract}
Ischemic stroke is one of the most common public health problems worldwide. The aim of the present study was to investigate the role of microRNA-19a (miR-19a) and its possible target genes in SK-N-SH cells subjected to oxygen-glucose deprivation/re-oxygenation (OGD/R) injury. SK-N-SH cells are a suitable model for host transfection. SK-N-SH cells were transfected with miR-19a mimic or inhibitor and PTEN-small interfering (si) RNA in order to alter the expression of miR-19a, PTEN and AKT. The expression changes in acute cerebral ischemic injury (ACII) were verified using RTqPCR and Western blotting. Expression changes and the association between miR-19a and PTEN following OGD/R were also assessed using a double luciferase analysis. In addition, cell viability and apoptosis were measured using an MTT and flow cytometry. miR-19a was downregulated; however, PTEN was markedly increased following OGD/R injury. miR-19a mimics increased cell viability, decreased cell apoptosis of SK-N-SH cells following OGD/R, which effects was similar to PTEN siRNA; however, miR-19a inhibitor had the opposite roles with miR-19a mimics. The present study provides novel information about the cell apoptosis and invasion mechanisms associated with the miR-19a/PTEN/AKT pathway and may present a potential therapeutic approach for OGD/R injury.
\end{abstract}

Key words: Oxygen-glucose deprivation/reoxygenation - microRNA-19a - PTEN — Neuroprotection

Abbreviations: ACII, acute cerebral ischemic injury; miR-19a, microRNA-19a; OGD/R, oxygen-glucose deprivation/reoxygenation; PTEN, phosphatase and tensin homolog deleted on chromosome 10.

\section{Introduction}

Stroke is a leading cause of mortality in a number of countries and the second leading cause of mortality in China (Hankey et al. 2010; Mortality and Causes of Death 2016).

\footnotetext{
* These authors participated equally to this study.

Correspondence to: Youtao Zhang, Department of Center for Clinical Laboratory, First Affiliated Hospital of Soochow University, Shizi Street Hospital District, No.188 Shizi Road, Suzhou, 215006, Jiangsu, People’s Republic of China.

E-mail: zhangytkh@163.com
}

Disability and consciousness disorders following stroke are a major burden for patients and their families. The majority of incidences of stroke are ischemic stroke (Tian et al. 2018). Ischemic injury to brain tissue following the inflammatory cascade response provoked by an oxygen-glucose deprivation/re-oxygenation typically causes ischemic brain damage and secondary brain injury (Wang et al. 2016). Despite developments in medical science, there is currently no effective therapeutic strategy for acute cerebral ischemic injury (ACII). It is therefore of great interest to develop novel treatments for ACII in order to reduce the morbidity and mortality. 
MicroRNAs (miRNAs/miRs) are small noncoding RNAs with length of 17-22 nucleotides. miRNAs are produced by a two-step pathway mediated by the RNaseIII enzymes Drosha and Dicer, which yield the mature miRNA molecules incorporating into a polyprotein complex called RNA-induced silencing complex and subsequent complete or incomplete binding to the 3'-untranslated region (UTR) of their respective target mRNAs that negatively regulate target gene expression at the post-transcriptional level by inhibiting translation and degrading target mRNAs (Bartel 2004; Bentwich et al. 2005). miRNAs have been suggested to play a key role in cerebral ischemic injury (Khoshnam et al. 2017b). miRNAs have been identified as important regulators of several biological processes, including cell differentiation, growth, proliferation, migration and apoptosis (Cho 2010; Aigner 2011; Rottiers et al. 2011). miR-19a is encoded by the oncogenic miR-17-92 cluster, which is considered to serve a role in a number of different cancers (Zhang et al. 2017). The oncogenic effect of the miR-17-92 cluster in B cell lymphoma has been demonstrated (Ventura et al. 2008; Mu et al. 2009) and several pro-apoptotic genes, including Phosphatase and tensin homolog deleted on chromosome 10 (PTEN), have been confirmed as target genes of miR-17-92 (O'Donnell et al. 2005; Novotny et al. 2007). However, the function and role of miR-19a in acute cerebral ischemic injury remain unclear. PTEN is a novel tumor suppressor gene whose double phosphatase activity has been identified in recent years (Stumpf and den Hertog 2016). It is considered to be one of the most important tumor suppressor genes along with p53, and is located on autosomes 10q23.3 (200 kb) (Fulci et al. 2000; Tate et al. 2009).

Dephosphorylating of protein kinase B (AKT) at Ser473 but not at Thr308 is mediated by one or both of the pleckstrin homology domain leucine-rich repeat protein phosphatase family of phosphatases (Crotty et al. 2013). Kroner et al. (2000) reported that the phosphorylation of Thr308 and Ser473 may be performed in either order independently of each other, although phosphorylation of Ser 473 is required to fully activate AKT. As such, the level of phosphorylated (p)-AKT (Ser473) is the primary marker of AKT activation and reflects the overall amount of p-AKT (Chua et al. 2009; Riaz et al. 2012). AKT, the serine-threonine kinase, is a key molecule that serves a role in the phosphoinositide (PI3K)/ AKT pathway and is able to inhibit apoptosis to promote cell survival (Ge et al. 2019). Previous studies have reported that apoptosis occurs in the peripheral penumbra of ischemia (Hou and MacManus 2002; Broughton et al. 2009) and pAKT may inhibit apoptosis. It has also been reported that the expression of $\mathrm{p}-\mathrm{AKT}$ is temporarily upregulated at the onset of focal cerebral ischemia and downregulated at $24 \mathrm{~h}$ following reperfusion; however, no significant changes in AKT expression were observed (Zhao et al. 2006). AKT phosphorylation promotes cell survival following cerebral ischemic insult via inducing the phosphorylation and subsequent inactivation of several pro-apoptotic proteins, including glycogen synthase kinase (GSK) $3 \beta$ (Endo et al. 2006). The $\mathrm{PI} 3 \mathrm{~K} / \mathrm{AKT}$ pathway mediates neuronal survival following cerebral ischemia and reperfusion (Endo et al. 2006; Zhu et al. 2013), which suggests that p-AKT308 and p-AKT473 may serve a role in cerebral ischemia-reperfusion injury.

Human SK-N-SH, a neuroblastoma cell line, is considered to be a core cell model of cerebrovascular tissues for in vitro assessments (Maggi et al. 1998). In order to assess the overall prognosis for brain tissue, these human neuroblastoma cells were selected for in vitro experiments in the present study. Cerebral infarction is caused by interrupted blood flow, leading to oxygen and glucose deprivation in the brain, which in turn results in infarction due to cell dysfunction and death (Verklan 2009; Pearson-Smith and Patel 2017). The oxygenglucose deprivation/re-oxygenation (OGD/R) cell model is a classical in vitro cell model of stroke (Irmady et al. 2014). The ultimate consequence of neuronal glucose deprivation is neuronal death by apoptosis or necrosis. Necrosis is a passive death process that occurs in cells that lack specificity, whereas apoptosis is a form of programmed cell death and is an active, regulated process (Gong et al. 2014). Apoptosis is associated with the aging process throughout life, making it is a focus of experimental research. In the present study, an OGD/R model was constructed based on SK-N-SH cells to simulate cerebral ischemia-reperfusion injury ( $\mathrm{Li}$ et al. 2017). The cell model was transfected with a miR-19a mimic or inhibitor and PTEN small interfering (si) RNA to investigate the potential role of miR-19a in OGD/R injury in vitro and to identify potential target genes of miR-19a, which may be associated with mechanisms of OGD/R injury and be potential targets for therapy.

\section{Materials and Methods}

\section{Ethics approval and consent to participate}

The present study protocol was approved by the Ethics Committee of the First Affiliated Hospital of Soochow University and carried out following the NIH guidelines. All patients provided written informed consent.

\section{Clinical experiments and grouping}

A total of 30 paired peripheral blood samples were collected from 20 patients with acute cerebral infarction (18 male, 12 female; mean age, 54 years; age range, 36-72 years) (cerebral infarction group) and 20 normal healthy volunteers (17 male, 13 female; mean age, 55 years; age range, 35-75 years) (normal group) at the First Affiliated Hospital of Soochow University (Suzhou, China) between June 2015 and 
June 2016. The National Institutes of Health (NIH) Stroke Scale system was used to evaluate the severity of ischemic stroke. Patients were excluded if any other central nervous system disease was identified. Venous blood samples $(5 \mathrm{ml})$ were drawn from all participants. The serum was isolated by centrifuging at $1,000 \times g$ at $4^{\circ} \mathrm{C}$ for $5 \mathrm{~min}$ and then stored in liquid nitrogen. Reverse transcription-quantitative polymerase chain reaction (RT-qPCR) was used to detect the expression of miR-19a in the samples.

\section{Cell lines and culture}

Human SK-N-SH cells lines were purchased from the American Type Culture Collection (Manassas, VA, USA) and cultured as a monolayer in $25-\mathrm{cm}^{2}$ flasks containing Dulbecco's Modified Eagle's Medium (DMEM; Invitrogen; Thermo Fisher Scientific, Inc., Waltham, MA, USA) supplemented with $10 \%$ fetal bovine serum (FBS; Gibco; Thermo Fisher Scientific, Inc.) and 1\% penicillin-streptomycin. All cells were maintained in an incubator containing $5 \% \mathrm{CO}_{2}$ at $37^{\circ} \mathrm{C}$ and underwent passage twice weekly.

\section{OGD/R model}

The normal cell culture medium was removed and SK-N-SH cells were washed three times with PBS. The culture medium was replaced with serum/glucose-free DMEM and cells were placed into a hypoxic chamber at $37^{\circ} \mathrm{C}\left(95 \% \mathrm{~N}_{2}\right.$ and $5 \% \mathrm{CO}_{2}$ ) for $16 \mathrm{~h}$. Cells in the Control group were cultured under normoxic conditions for the same time. All cells were subsequently re-perfused for $12 \mathrm{~h}$ under normal culture conditions at $37^{\circ} \mathrm{C}\left(95 \% \mathrm{O}_{2}\right.$ and $\left.5 \% \mathrm{CO}_{2}\right)$.

\section{RNA isolation}

Total RNA was isolated from the cultured cells using TRIzol according to the manufacturer's protocol (Invitrogen; Thermo Fisher Scientific, Inc.). The concentration and purity of the RNA was detected using a NanoDrop ${ }^{\text {Tx }}$ Spectrophotometer 2000 (Thermo Fisher Scientific, Inc., Pittsburgh, PA, USA) at $260 \mathrm{~nm}$.

\section{$R T-q P C R$}

The expression of miR-19a in the Control and OGD/R groups was determined using RT-qPCR, performed using TaqMan miRNA assays (Thermo Fisher Scientific, Inc.) according to the manufacturer's protocol. Total RNA was extracted using TRIzol (Invitrogen; Thermo Fisher Scientific, Inc.) and cDNA synthesis was performed with a reverse transcription kit (Promega Corporation, Madison, WI, USA). The PCR primers used were as follows: PTEN, forward 5'-TTTGAAGACCATAACCCACCAC-3' and reverse
5'-ATTACACCAGTTCGTCCCTTTC-3'; and GAPDH, forward 5'-CTGGGCTACACTGAGCACC-3' and reverse 5'-AAGTGGTCGTTGAGGGCAATG-3'. The thermocycling conditions were as follows: Initial denaturation for $10 \mathrm{~min}$ at $95^{\circ} \mathrm{C}$ followed by 40 cycles at $95^{\circ} \mathrm{C}$ for $15 \mathrm{~s}$ and $60^{\circ} \mathrm{C}$ for $1 \mathrm{~min}$. All RT-PCR reactions were performed 3 times. qPCR was performed with SYBR Premix Ex Taq II (Takara Bio, Inc., Otsu, Japan) using a Light Cycler 480 system (Roche Diagnostics, Basel, Switzerland). The $2^{-\Delta \Delta \mathrm{Cq}}$ method was used to quantify the relative RNA expression (Livak and Schmittgen 2001). U6 was used as a reference gene for miRNA quantification, and GAPDH was used as a reference gene for proteins quantification.

\section{Transfection and grouping}

Following OGD/R, SK-N-SH cells were transfected with miR-19a mimic, miR-19a inhibitor, PTEN siRNA or the corresponding negative control (NC) using DharmaFect 2000 Transfection Reagent (Thermo Fisher Scientific, Inc.) as previously described (Bentwich et al. 2005). The SK-N-SH cells were collected $24 \mathrm{~h}$ following transfection and groups were designed as follows: OGD/R+NC group, OGD/R+miR19a mimic group, OGD/R+miR-19a inhibitor group and OGD/R+PTEN siRNA group.

\section{Measurement of cell viability}

The viability of SK-N-SH cells was measured with an MTT assay. The MTT working solution (1:10 dilution of the $5 \mathrm{mg} / \mathrm{ml}$ stock; Promega Corporation, Madison, WI, USA) was filtered through a $0.22-\mu \mathrm{m}$ filter and stored at $4^{\circ} \mathrm{C}$. SK$\mathrm{N}-\mathrm{SH}$ cells were washed with warm RPMI-1640 medium (Thermo Fisher Scientific, Inc.) without phenol red, following which the cells were seeded at a density of $8 \times 10^{3}$ cells/well in 96-well plates containing DMEM with $10 \%$ fetal bovine serum, and cultured at $37^{\circ} \mathrm{C}$ for $12 \mathrm{~h}$ in a $5 \% \mathrm{CO}_{2}$ incubator. A total of $20 \mu \mathrm{l}$ MTT working solution was added into each experimental well, following which plates were incubated at $37^{\circ} \mathrm{C}$ for a further $2 \mathrm{~h}$. Then MTT solution was removed, and dimethyl sulfoxide was added to each well to dissolve the purple formazan. The absorbance of each well was read at a wavelength of $570 \mathrm{~nm}$ using a microplate reader. Absorbance was assumed to be directly proportional to the number of surviving cells and the viability of the control group was assumed to be $100 \%$. A total of 4 independent experiments were performed, in quadruplicate.

\section{Evaluation of miR-19a and PTEN using a double-luciferase targeting assay}

miR-19a has previously been demonstrated to target PTEN. For the luciferase reporter assays, a pGL3 Firefly 
Luciferase reporter vector (Promega Corp., Madison, WI, USA), with the 3'UTR fragment of human PTEN cDNA containing the putative target site for miR-19a and serving as an endogenous transfection control. A QuickChange Multi Site-Directed Mutagenesis kit (Stratagene; Agilent Technologies, Inc., Santa Clara, CA, USA) was used to mutate the human-miR-19a binding site in the PTEN 3'-UTR. The mutated (MUT) 3'-UTR sequence was used to create a luciferase plasmid, luc-PTEN-MUT, without a complementary miR-19a binding sequence. The SK-NSH cells were transfected with the PTEN 3'UTR reporter or the MUT 3'UTR (30 ng/Ml) using Lipofectamine $2000^{\circledR}$ reagent (Invitrogen; Thermo Fisher Scientific, Inc.). Furthermore, a Renilla luciferase construct was also co-transfected to normalize transfection efficiency. At $24 \mathrm{~h}$ post transfection, a dual-luciferase assay (Promega Corp.) was performed to assess the relative luciferase activities under a FL500 microplate fluorescence reader (BioTek Instruments, Inc., Winooski, VT, USA), according to the manufacturer's protocol. A minimum of three independent experiments was performed as previously described (Bartel 2004).

\section{Measurement of SK-N-SH cell apoptosis using flow cytometry}

The SK-N-SH cells were digested with Trypsin-EDTA (0.25\%), phenol red (Thermo Fisher Scientific, Inc.) and $1 \times 10^{5}$ cells $/ \mathrm{ml}$ were seeded in 6 -well plates. Apoptotic cells were detected using an Annexin V-fluorescein isothiocyanate (FITC)/PI flow cytometry assay (BD Biosciences) and Cell Quest Software (version 3.1) according to the manufacturer's protocol. In brief, cells transfected with PTEN siRNA and miR-19a mimic for $48 \mathrm{~h}$ were fixed in pre-cooled $70 \%$ ethanol at $4^{\circ} \mathrm{C}$ overnight. The cells were harvested, washed twice with cold PBS and suspended in cold Annexin V-FITC binding buffer. Following the addition of $5 \mu \mathrm{l}$ Annexin V-FITC and $5 \mu \mathrm{lPI}$, the cells were incubated in the dark at room temperature for $30 \mathrm{~min}$. Each sample was subsequently incubated with $400 \mu \mathrm{l}$ binding buffer. The samples were analyzed using the FACSCalibur flow cytometer (BD Biosciences). A total of three independent experiments were performed.

\section{Western blotting}

SK-N-SH cells were transfected with an NC, miR-19a mimic, miR-19a inhibitor or PTEN siRNA. At $48 \mathrm{~h}$ after miRNA transfection, the SK-N-SH cells were homogenized in a lysis buffer $(50 \mathrm{mM}$ Tris- $\mathrm{HCl}, \mathrm{pH} 8.0 ; 10 \mathrm{mM}$ $\mathrm{NaCl}$; 1 MM DTT; 2mM EDTA; 1\% NP-40; 0.1\% SDS and protease and phosphatase inhibitor cocktails) and total protein was extracted using radio immunoprecipitation assay buffer (Thermo Fisher Scientific, Inc.). The protein concentration was measured using a BCA kit (Bio-Rad Laboratories, Inc., Hercules, CA, USA). For Western blot analysis the protein lysates $(20 \mu \mathrm{g} / \mathrm{lane})$ were separated on $10 \%$ SDS-PAGE and transferred to polyvinylidene difluoride membranes. Then $10 \%$ skimmed milk was used to block the membranes for $1 \mathrm{~h}$ at room temperature. The membrane was probed with primary antibodies against PTEN (ab32199; 1:10,000), AKT (ab8805; 1:500), p-AKT308 (ab38449; 1:500) or p-AKT473 (ab8933; 1:500; all Abcam, Cambridge, UK). GAPDH (ab8245; 1:1,000, Abcam) overnight at $4^{\circ} \mathrm{C}$. GAPDH served as the loading control. The membranes were subsequently incubated with horseradish peroxidase-conjugated secondary antibodies (1:1000, Santa Cruz Biotechnology, Santa Cruz, CA, USA) at room temperature for $2 \mathrm{~h}$. The protein bands were scanned using a Chemi Doc XRS+ Imaging System and quantified using Quantity One v4.6.2 software (both Bio-Rad Laboratories, Inc.).

\section{Statistical analysis}

Data are expressed as the mean \pm standard error of the mean. Differences between the means were analyzed using a Student's $t$-test. Differences between multiple groups were assessed by one-way analysis of variance followed by the Dunnett's post-hoc test. Each experiment was repeated $\geq 3$ times. Calculations were performed using Excel software (Microsoft Corporation, Redmond, VA, USA) and the data were plotted using GraphPad Prism 6.0 software (GraphPad Software, Inc., La Jolla, CA, USA). A value $p<$ 0.05 was considered to indicate a statistically significant difference.

\section{Results}

miRNA-19a expression in peripheral blood samples from patients with acute cerebral infarction and normal healthy volunteers

RT-qPCR results indicated that the expression of miR-19a was significantly downregulated in the patients with acute cerebral ischemic injury when compared with that in normal healthy volunteers (Fig. 1A; $p<0.01$ ).

\section{Expression of miRNA-19a and PTEN in SK-N-SH cells following $O G D / R$}

To confirm the differential expression of miRNA-19a following OGD/R, the expression of miRNA-19a and PTEN was examined using RT-qPCR and Western blotting. The results revealed that miRNA-19a mRNA was significantly 

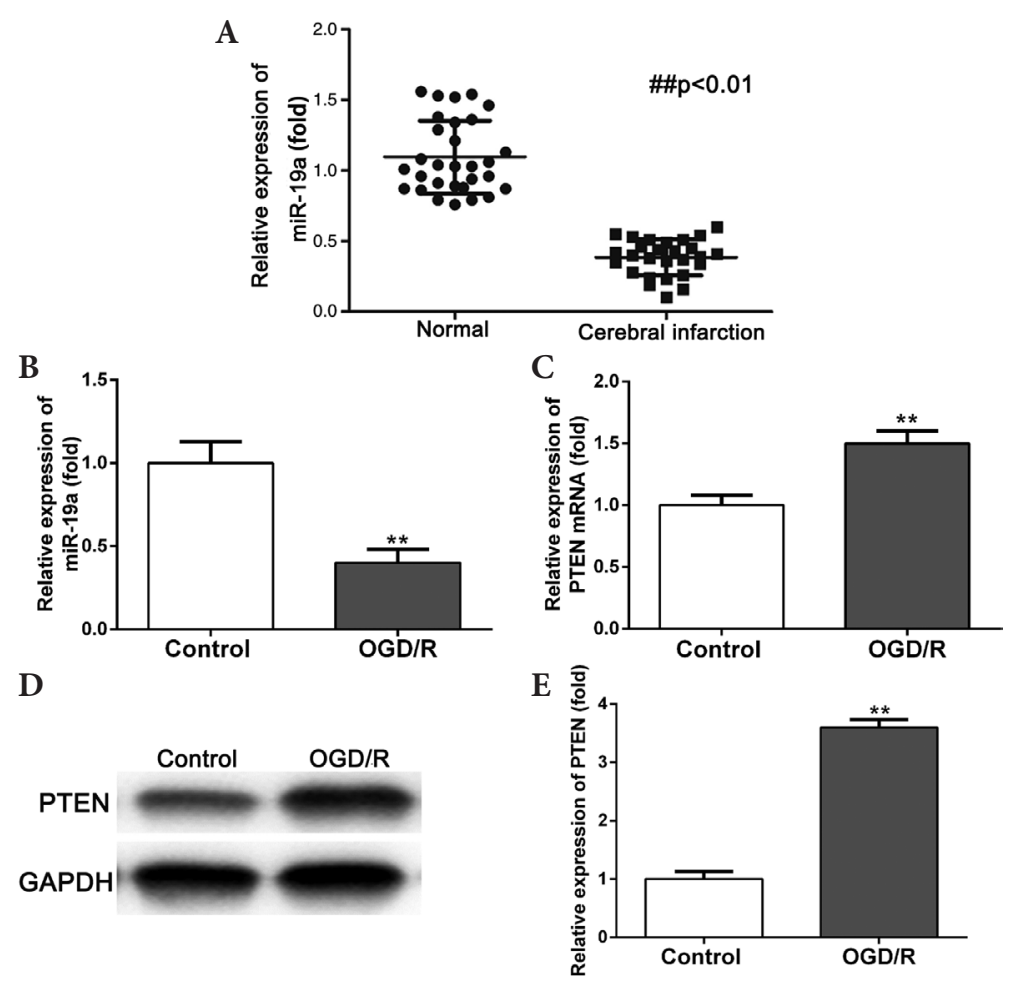

Figure 1. Comparison of miR-19a expression in peripheral blood samples from the normal healthy volunteers and the patients with acute cerebral ischemic injury (A), and in the SK-H-NH cells of Control and OGD/R treatment groups (B). The level of PTEN mRNA $(\mathbf{C})$ and the protein expression of PTEN (D) in the Control and OGD/R groups. E. The relative protein levels of PTEN in the Control and OGD/R groups. ${ }^{\# \#} p<0.01 v$ s. Normal healthy volunteers. ${ }^{* *} p<0.01$ $v s$. Control group. miR, microRNA; OGD/R, oxygenglucose deprivation/reperfusion. downregulated in the OGD/R group compared with the Control group (Fig. 1B; $p<0.01$ ). However, the expression of PTEN mRNA was significantly elevated in the OGD/R group compared with the Control group (Fig. 1C; $p<0.01$ ). Similarly, Western blotting revealed that the expression of PTEN protein was significantly upregulated in the OGD/R group compared with the Control (Fig. 1D and E; $p<0.01$ ), which is consistent with the results of RT-qPCR.

Expression of miRNA-19a and PTEN in transfected SK-N-SH cells following $O G D / R$

Following OGD/R and transfection with miR-19a mimic/ inhibitor, PTEN siRNA or NC, the expression of miRNA19a and PTEN in SK-N-SH cells was assessed using RT-qPCR and Western blotting. The results of RT-qPCR revealed that miRNA-19a was upregulated in the miR-19a mimic and PTEN-siRNA groups compared with the NC group (Fig. 2A; $p<0.01$ ), while miRNA-19a expression was downregulated in the miR-19a inhibitor group (Fig. 2A; $p<0.05)$. The expression of PTEN mRNA was increased in the miR-19a inhibitor group compared with the NC group (Fig. 2B; $p<0.05$ ); however, PTEN mRNA expression was reduced in the miR-19a mimic and PTEN-siRNA groups compared with the NC group (Fig. $2 \mathrm{~B} ; p<0.05$ ). The expression of PTEN at the protein level was detected using Western blotting (Fig. 2C) and the results demonstrated that PTEN protein expression was significantly upregulated in the miR-19a inhibitor group compared with the NC group (Fig. 2D; $p<0.01$ ).

\section{PTEN is a direct target of $m i R-19 a$}

A schematic illustration of miR-19a binding to the wildtype (WT) PTEN 3'UTR is presented in Fig. 3A. Cells co-transfected with miR-19a mimic and PTEN 3'UTRWT exhibited less cell luciferase activity compared with those co-transfected with NC+PTEN 3'UTR-WT or Control+PTEN 3'UTR-WT (Fig. 3A; $p<0.05$ ). No significant differences in luciferase activity were observed in cells co-transfected with miR-19a+PTEN 3'UTR-MUT, NC+PTEN 3'UTR-MUT or Control+PTEN 3'UTR-MUT (Fig. 3A). These results suggest that PTEN is negatively regulated by miR-19a.

\section{miRNA-19a affects cell proliferation of SK-N-SH cells following $O G D / R$}

MTT results demonstrated that optical absorption in the miR-19a inhibitor group was significantly decreased compared with the NC group (Fig. 3B; $p<0.05$ or $p<0.01$ ). By contrast, transfection with miR-19a mimic or PTEN-siRNA significantly increased cell viability compared with NC (Fig. $3 \mathrm{~B} ; p<0.05$ or $p<0.01)$. 
A

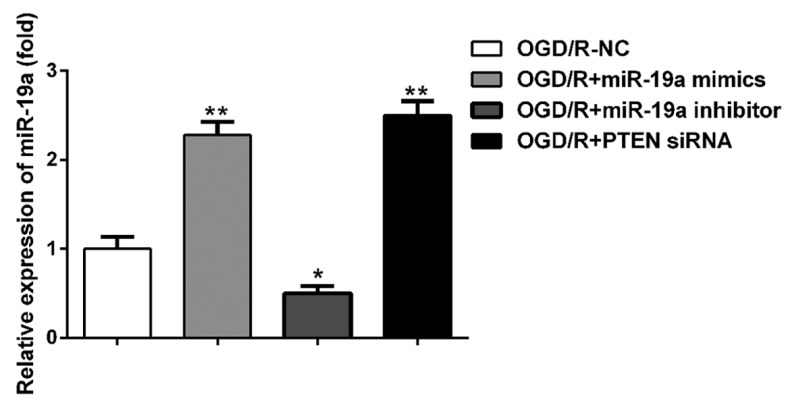

B

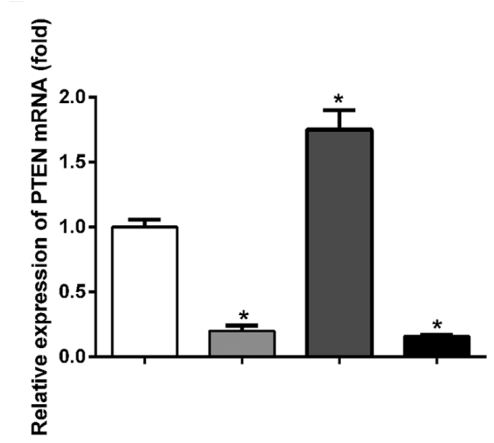

C

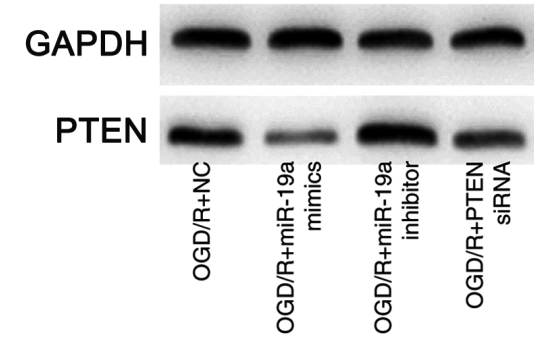

D

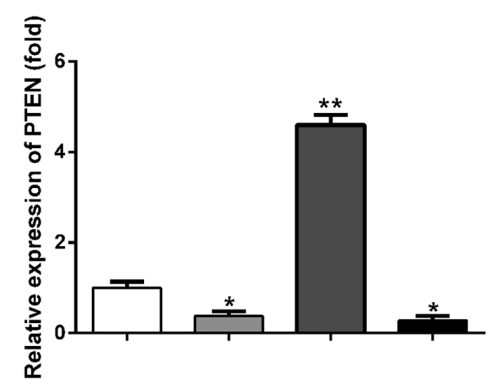

Figure 2. Expression of miR-19a (A) and PTEN (B) in SK-H$\mathrm{NH}$ cells subjected to OGD/R treatment and transfected with miR-19a mimic, miR-19a inhibitor or PTEN siRNA. Protein expression (C) and relative protein levels (D) of PTEN in SK$\mathrm{H}-\mathrm{NH}$ cells transfected with miR-19a mimic was detected using Western blotting miR-19a inhibitor or PTEN siRNA after $\mathrm{OGD} / \mathrm{R} .{ }^{\star} p<0.05$ and ${ }^{*} p<0.01 v s$. the $\mathrm{OGD} / \mathrm{R}+\mathrm{NC}$ group. miR, microRNA; OGD/R, oxygen-glucose deprivation/reperfusion; NC, negative control.
miRNA-19a overexpression affects the cell apoptosis of SK-N-SH cells following OGD/R

Flow cytometry was performed to investigate the effects of exogenous miR-19a on cell apoptosis. The number of apoptotic cells was significantly higher in the miR-19a inhibitor group compared with the NC group (Fig. $4 ; p<0.05$ ). Conversely, the number of apoptotic cells was significantly reduced in the miR-19a mimic and PTEN-siRNA groups (Fig. $4 ; p<0.01$ ). This suggests that miR-19a upregulation or PTEN knockdown is able to promote cell cycle progression and suppress apoptosis in SK-N-SH cells.

Expression of AKT, p-AKT308 and p-AKT473 in SK-N-SH cells following $O G D / R$

The expression of p-AKT308 and p-AKT473 in the miR19a mimic and PTEN-siRNA groups was significantly

A
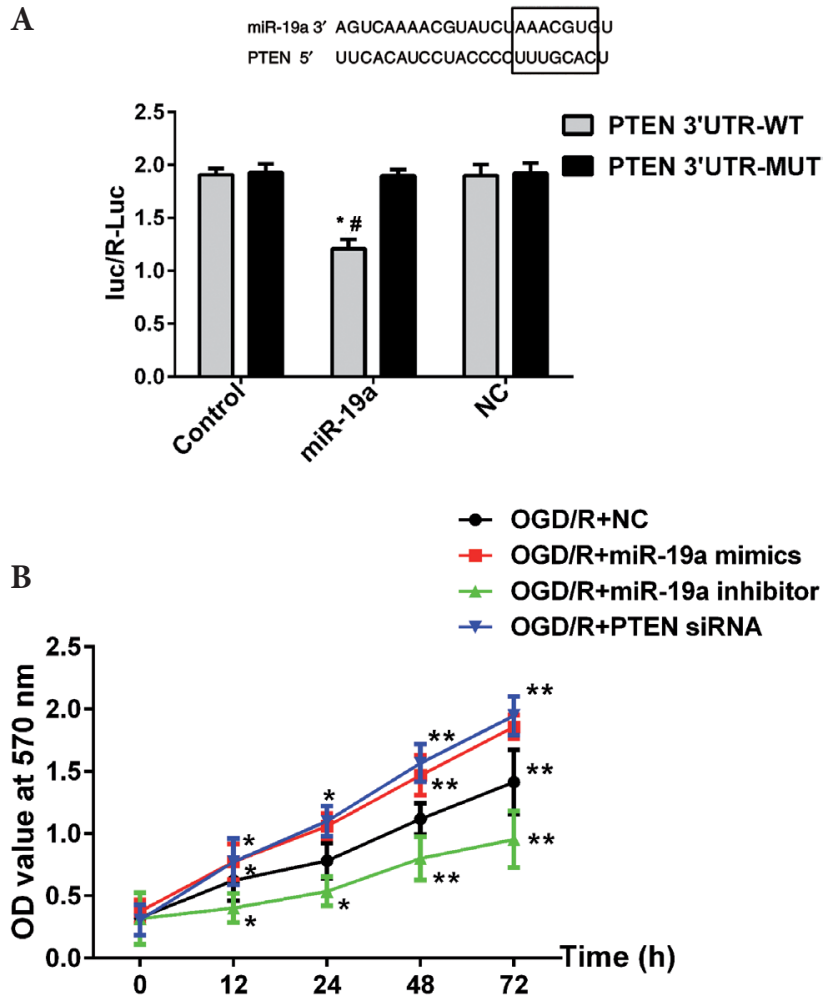

Figure 3. miR-19a targets PTEN. A. Putative binding of miR-19a to the PTEN gene 3'-UTR. SK-H-NH cells were co-transfected with luciferase plasmids containing the luc-PTEN-WT or luc-PTENMUT and the relative luciferase activities in different groups were determined. ${ }^{*} p<0.05 v s$. NC+PTEN 3'UTR-WT and ${ }^{\#} p<0.05$ $v$ s. Control+PTEN 3'UTR-WT group. B. MTT growth assays. ${ }^{*} p<$ $0.05,{ }^{* *} p<0.01 v$ s. OGD/R+NC group. UTR, untranslated region; WT, wild type; MUT, mutant; siRNA, small interfering RNA; OD, optical density. For more abbreviations, see Fig. 2. 
increased compared with the NC group (Fig. 5A and B; $p<0.01$ ). Similarly, the expression of p-AKT308 and p-AKT473 in the miR-19a inhibitor group was higher compared with the NC group (Fig. 5A and B; $p<0.05$ ). No significant differences were observed in AKT between groups (Fig. 5C).

\section{Discussion}

Ischemic stroke is the third leading cause of death in developing countries and is a leading cause of disability in developing countries and China (Norrving and Kissela 2013). The morbidity of stroke has increased with increased life expectancy. There are multiple risk factors for stroke, including genetics and the environment (Vinters et al. 2018). However, the pathogenesis of stroke remains poorly understood. It is therefore important to explore the pathogenic and protective mechanisms associated with stroke.

In recent years, it has been reported that cerebral ischemic injury may alter the expression of several miRNAs in ischemic brain tissue (Khoshnam et al. 2017a). miRNAs serve roles in development, differentiation and apoptosis in the nervous system (Nowak and Michlewski 2013; Tielking et al. 2019), as well as a number of nervous system diseases, including cerebral cancer, Alzheimer's disease, Parkinson's disease and ischemic stroke (Kalani et al. 2013; Roth et al. 2016; Quinlan et al. 2017). The level of miRNAs in cerebral ischemic tissue and the peripheral blood may be associated with the conditions of ischemic stroke (Khoshnam et al. 2017a). In addition, cerebral ischemia is known to alter the miRNA profile in a spatial-temporal manner (Zou et al. 2019). The present study demonstrated that there was a marked reduction in miR-19a expression in the blood of patients who had experienced cerebral infarction. The results of the present study demonstrated that miR-19a was decreased in SK-H-NH cells subjected to OGD/R compared with normal control cells. Following OGD/R, miR-19a levels were decreased in SK-H-NH cells with a corresponding increase in PTEN expression. This suggests that miR-19a may act as a significant regulator of PTEN expression and so may have potential as a therapeutic agent for reducing neuronal apoptosis following ischemic injury. Furthermore, PTEN protein expression was significantly decreased in SK-N-SH cells transfected with miRNA-19a mimic or PTEN siRNA, whereas transfection with miRNA-19a inhibitor resulted in increased PTEN protein expression. These results suggest that miRNA-19a may serve a crucial role in the response to $\mathrm{OGD} / \mathrm{R}$ and the regulation of PTEN.

In the present study, cell apoptosis was assessed in SK-HNK cells following OGD/R treatment using flow cytometry. The results demonstrated that miR-19a knockdown accelerates cell apoptosis and enhances OGD/R injury, whereas miR-19a overexpression reduces cell apoptosis and has a protective effect. Collectively, these findings indicate that miR-19a upregulation may serve an important neuroprotective role in OGD/R injury. Furthermore, transfection with miR-19a mimic reduced luciferase activity in cells expressing WT but not MUT vectors, while overexpression or suppression of miR-19a resulted in a decrease or increase in PTEN expression during $\mathrm{OGD} / \mathrm{R}$ injury, respectively. These data suggest that PTEN is a direct target gene of miR-19a. Ap-
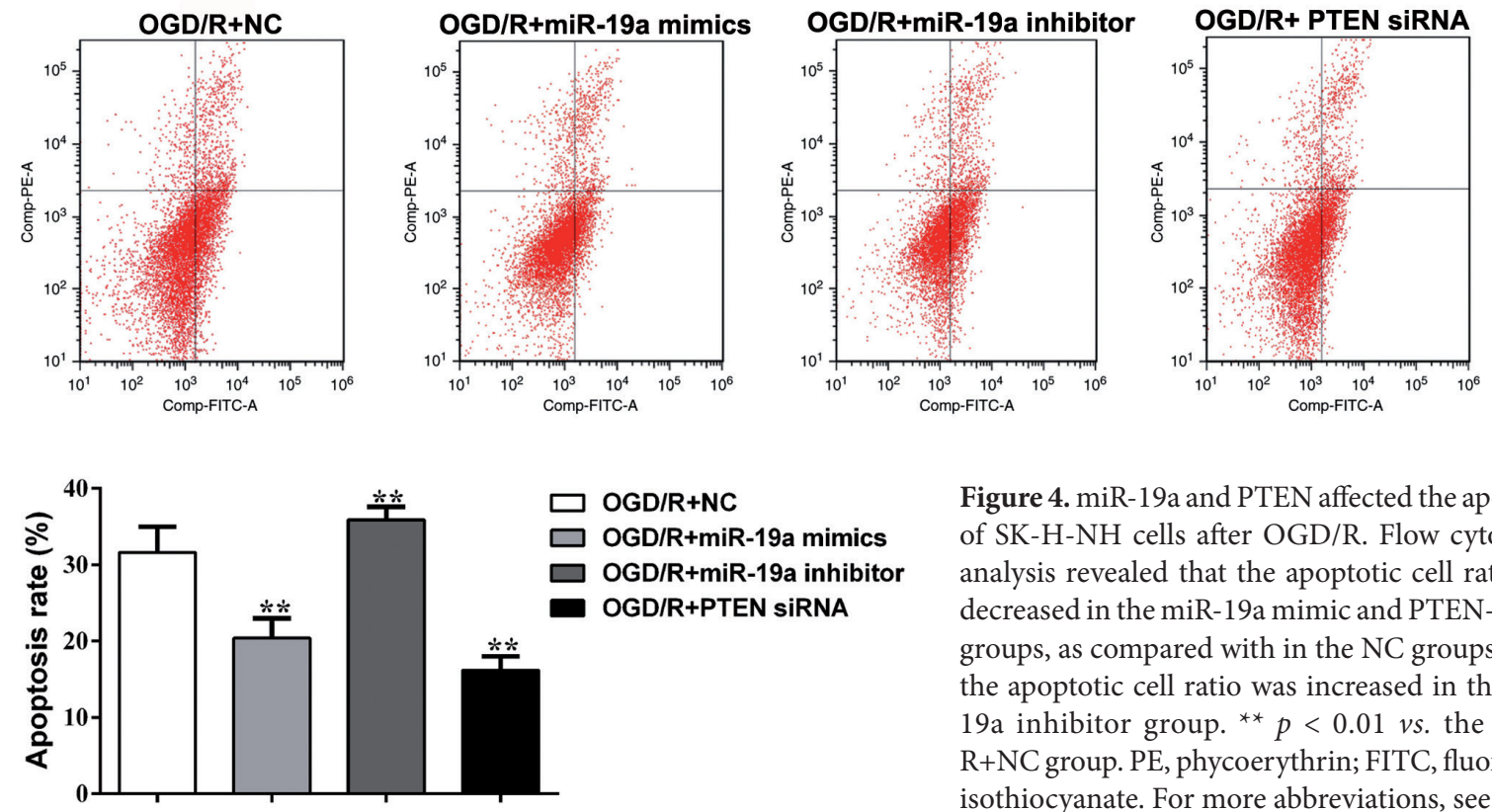

Figure 4. miR-19a and PTEN affected the apoptosis of SK-H-NH cells after OGD/R. Flow cytometry analysis revealed that the apoptotic cell ratio was decreased in the miR-19a mimic and PTEN-siRNA groups, as compared with in the NC groups, while the apoptotic cell ratio was increased in the miR19a inhibitor group. ${ }^{* *} p<0.01 v s$. the OGD/ $\mathrm{R}+\mathrm{NC}$ group. PE, phycoerythrin; FITC, fluorescein isothiocyanate. For more abbreviations, see Fig. 3. 
A

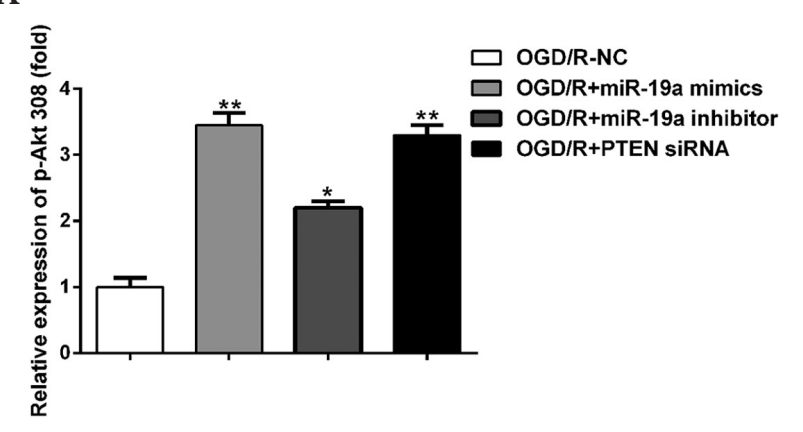

B

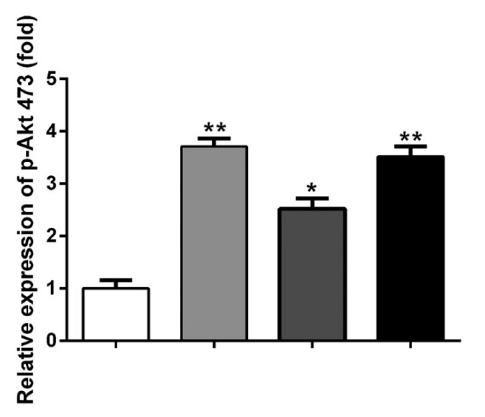

C

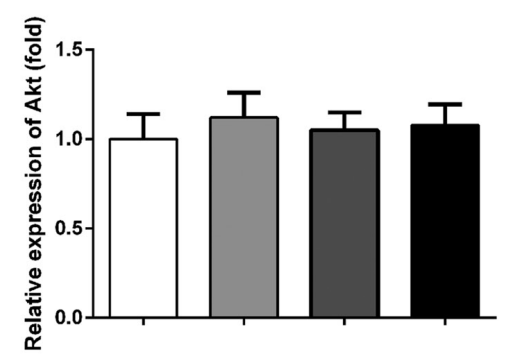

D

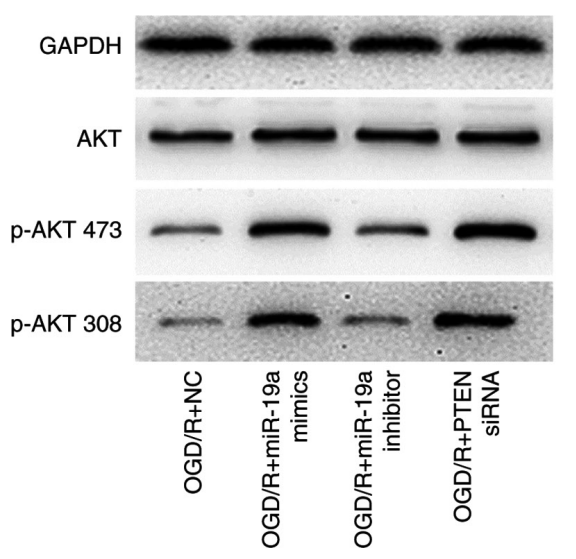

Figure 5. Relative levels of p-AKT 308 (A), p-AKT 473 (B) and $\mathrm{AKT}(\mathrm{C})$ in the OGD/R+NC, OGD/R+miR-19a inhibitor, OGD/ $\mathrm{R}+$ miR-19a mimic and OGD/R+PTEN siRNA groups were determined using Western blotting (D). ${ }^{*} p<0.05$ and ${ }^{*} p<0.01 v s$. the OGD/R +NC group. p, phosphorylated; AKT, protein kinase B. For more abbreviations, see Fig. 3 . optosis and autophagy programmed cell death are the main routes by which nerve cell death occurs in cerebral ischemiareperfusion injury (Wei et al. 2016) and are regulated by apoptosis-related genes and their products.

The results of the present study suggest that transfection with miR-19a inhibitor results in decreased viability of SK$\mathrm{N}-\mathrm{SH}$ cells and induces cell death. As a further experiment, we focused on the targets of miR-19a, since the translation of the mRNA targets can be inhibited or the mRNAs degraded by miRNA to prevent their functions. In order to accurately predict the target genes of miR-19a, we evaluated miRecords, and identified that PTEN could be used as a target gene for the subsequent experiments. The results of Western blotting indicate that miR-19a overexpression decreased the expression of PTEN protein, whereas miR-19a knockdown had the opposite effect. In addition, the phosphorylation of AKT is a primary target of PTEN. The results of the present study indicate that the expression levels of p-AKT308 and p-AKT473 are increased by miR-19a overexpression and decreased by the miR-19a inhibition. Luciferase reporter assays also confirmed that PTEN is a direct target of miR-19a in SK-N-SH cells. SK-N-SH cells transfected with miR-19a inhibitor underwent OGD/R, which decreased the expression of miR-19a and upregulated the expression of its target gene PTEN, as well as increasing the expression of p-AKT308 and p-AKT473 protein. However, transfection with miR-19a mimic or PTEN siRNA suppressed the expression of PTEN while upregulating p-AKT308 and p-AKT473. These results suggest that miR-19a-PTEN-p-AKT is an important pathway in OGD/R injury. These findings suggest that miR-19a overexpression protects SK-H-NH cells from OGD/R injury by downregulating PTEN expression as a neuroprotective strategy. MiR-19a mimics may have potential as neuroprotective pharmacological agents for the treatment of cerebral ischemic injury. Further investigation into the opposing functions of miR-19a and PTEN may contribute to our understanding of the complex regulatory mechanisms of miRNAs in OGD/R injury.

In conclusion, the present study revealed that $\mathrm{miR}-19 \mathrm{a}$ targets PTEN mRNA directly and functions as an oncogene via the PTEN/AKT signaling pathway in OGD/R injury. However, it has certain limitations. The molecular mechanism of miR-19a in regulating OGD/R injury was studied in vitro only; further in vivo studies are required to verify these results. In addition, miRNAs have a complex mechanism of action in the regulation of human diseases, and miR19a has a large number of target genes. The present study only investigated the role of miR-19a in targeting PTEN in OGD/R injury; whether other pathways or mechanisms are associated with OGD/R injury requires further study.

Conflict of interests. The authors declare that they have no competing interests. 


\section{References}

Aigner A (2011): MicroRNAs (miRNAs) in cancer invasion and metastasis: therapeutic approaches based on metastasis-related miRNAs. J. Mol. Med. 89, 445-457 https://doi.org/10.1007/s00109-010-0716-0

Bartel DP (2004): MicroRNAs: genomics, biogeness, mechanism, and function. Cell 116, 281-297 https://doi.org/10.1016/S0092-8674(04)00045-5

Bentwich I, Avniel A, Karov Y, Aharonov R, Gilad S, Barad O, Barzilai A, Einat P, Einav U, Meiri E, et al. (2005): Identification of hundreds of conserved and nonconserved human microRNAs. Nat. Genet. 37, 766-770 https://doi.org/10.1038/ng1590

Broughton BR, Reutens DC, Sobey CG (2009): Apoptotic mechanisms after cerebral ischemia. Stroke 40, e331-339 https://doi.org/10.1161/STROKEAHA.108.531632

Cho WC (2010): MicroRNAs: potential biomarkers for cancer diagnosis, prognosis and targets for therapy. Int. J. Biochem. Cell. Biol. 42, 1273-1281 https://doi.org/10.1016/j.biocel.2009.12.014

Chua BT, Gallego-Ortega D, Ramirez de Molina A, Ullrich A, Lacal JC, Downward J (2009): Regulation of Akt(ser473) phosphorylation by choline kinase in breast carcinoma cells. Mol. Cancer 8, 131 https://doi.org/10.1186/1476-4598-8-131

Crotty TM, Nakano T, Stafforini DM, Topham MK (2013): Diacylglycerol kinase delta modulates Akt phosphorylation through pleckstrin homology domain leucine-rich repeat protein phosphatase 2 (PHLPP2). J. Biol. Chem. 288, 1439-1447 https://doi.org/10.1074/jbc.M112.407379

Endo H, Nito C, Kamada H, Nishi T, Chan PH (2006): Activation of the Akt/GSK3beta signaling pathway mediates survival of vulnerable hippocampal neurons after transient global cerebral ischemia in rats. J. Cereb. Blood Flow Metab. 26, 1479-1489 https://doi.org/10.1038/sj.jcbfm.9600303

Fulci G, Labuhn M, Maier D, Lachat Y, Hausmann O, Hegi ME, Janzer RC, Merlo A, Van Meir EG (2000): p53 gene mutation and ink4a-arf deletion appear to be two mutually exclusive events in human glioblastoma. Oncogene 19, 3816-3822 https://doi.org/10.1038/sj.onc.1203700

Ge JC, Wang YX, Chen ZB, Chen DF (2019): Integrin alpha 7 correlates with poor clinical outcomes, and it regulates cell proliferation, apoptosis and stemness via PTK2-PI3K-Akt signaling pathway in hepatocellular carcinoma. Cell. Signal. 66, 109465 https://doi.org/10.1016/j.cellsig.2019.109465

Gong J, Kumar SA, Graham G, Kumar AP (2014): FLIP: molecular switch between apoptosis and necroptosis. Mol. Carcinog. 53, 675-685 https://doi.org/10.1002/mc.22027

Hankey GJ, Wong KSL, Chankrachang S, Chen C, Crimmins D, Frayne J, Kim JS, Li Y, Liou CW, Merican JS, et al. (2010): Management of cholesterol to reduce the burden of stroke in Asia: consensus statement. Int. J. Stroke 5, 209-216 https://doi.org/10.1111/j.1747-4949.2010.00429.x

Hou ST, MacManus JP (2002): Molecular mechanisms of cerebral ischemia-induced neuronal death. Int. Rev. Cytol. 221, 93-148 https://doi.org/10.1016/S0074-7696(02)21011-6
Irmady K, Jackman KA, Padow VA, Shahani N, Martin LA, Cerchietti L, Unsicker K, Iadecola C, Hempstead BL (2014): Mir-592 regulates the induction and cell death-promoting activity of p75NTR in neuronal ischemic injury. J. Neurosci. 4, 3419-3428 https://doi.org/10.1523/JNEUROSCI.1982-13.2014

Kalani A, Kamat PK, Tyagi SC, Tyagi N (2013): Synergy of homocysteine, microRNA, and epigenetics: a novel therapeutic approach for stroke. Mol. Neurobiol. 48, 157-168 https://doi.org/10.1007/s12035-013-8421-y

Khoshnam SE, Winlow W, Farbood Y, Moghaddam HF, Farzaneh M (2017a): Emerging roles of microRNAs in ischemic stroke: as possible therapeutic agents. J. Stroke 19, 166-187 https://doi.org/10.5853/jos.2016.01368

Khoshnam SE, Winlow W, Farzaneh M (2017b): The interplay of microRNAs in the inflammatory mechanisms following ischemic stroke. J. Neuropathol. Exp. Neurol. 76, 548-561 https://doi.org/10.1093/jnen/nlx036

Kroner C, Eybrechts K, Akkerman JW (2000): Dual regulation of platelet protein kinase B. J. Biol. Chem. 275, 27790-27798 https://doi.org/10.1074/jbc.M000540200

Li SH, Chen L, Pang XM, Su SY, Zhou X, Chen CY, Huang LG, Li JP, Liu JL (2017): Decreased miR-146a expression in acute ischemic stroke directly targets the Fbxl10 mRNA and is involved in modulating apoptosis. Neurochem. Int. 107, 156-167 https://doi.org/10.1016/j.neuint.2017.01.011

Livak KJ, Schmittgen TD (2001): Analysis of relative gene expression data using real-time quantitative PCR and the 2(-Delta Delta C(T)). Methods 25, 402-408 https://doi.org/10.1006/meth.2001.1262

Maggi R, Poletti A, Casulari LA, Pimpinelli F, Piva F, Zanisi MR, Martini L (1998): Effects and metabolism of steroid hormones in human neuroblastoma cells. Steroids 63, 257-262 https://doi.org/10.1016/S0039-128X(98)00038-5

Mortality GBD, Causes of Death Collaborators (2016): Global, regional, and national life expectancy, all-cause mortality, and cause-specific mortality for 249 causes of death, 1980-2015: a systematic analysis for the Global Burden of Disease Study 2015. Lancet 388, 1459-1544 https://doi.org/10.1016/S0140-6736(16)31012-1

Mu P, Han YC, Betel D, Yao E, Squatrito M, Ogrodowski P, de Stanchina E, D'Andrea A, Sander C, Ventura A (2009): Genetic dissection of the miR-17 92 cluster of microRNAs in Mycinduced B-cell lymphomas. Genes Dev. 23, 2806-2811 https://doi.org/10.1101/gad.1872909

Norrving B, Kissela B (2013): The global burden of stroke and need for a continuum of care. Neurology 80, S5-12 https://doi.org/10.1212/WNL.0b013e3182762397

Novotny GW, Sonne SB, Nielsen JE, Jonstrup SP, Hansen MA, Skakkebaek NE, Rajpert-De Meyts E, Kjems J, Leffers H (2007): Translational repression of E2F1 mRNA in carcinoma in situ and normal testis correlates with expression of the miR-17-92 cluster. Cell Death Differ. 14, 879-882 https://doi.org/10.1038/sj.cdd.4402090

Nowak JS, Michlewski G (2013): miRNAs in development and pathogenesis of the nervous system. Biochem. Soc. Trans. 41, 815-820 https://doi.org/10.1042/BST20130044 
O'Donnell KA, Wentzel EA, Zeller KI, Dang CV, Mendell JT (2005): c-Myc-regulated microRNAs modulate E2F1 expression. Nature $435,839-843$ https://doi.org/10.1038/nature03677

Pearson-Smith JN, Patel M (2017): Metabolic dysfunction and oxidative stress in epilepsy. Int. J. Mol. Sci. 18, e2365 https://doi.org/10.3390/ijms18112365

Quinlan S, Kenny A, Medina M, Engel T, Jimenez-Mateos EM (2017): MicroRNAs in neurodegenerative diseases. Int. Rev. Cell Mol. Biol. 334, 309-343 https://doi.org/10.1016/bs.ircmb.2017.04.002

Riaz A, Zeller KS, Johansson S (2012): Receptor-specific mechanisms regulate phosphorylation of AKT at Ser473: role of RICTOR in betal integrin-mediated cell survival. PLoS One 7, e32081 https://doi.org/10.1371/journal.pone.0032081

Roth SA, Knutsen E, Fiskaa T, Utnes P, Bhavsar S, Hald ØH, Løkke C, Mestdagh P, Johansen SD, Flægstad T, Einvik C (2016): Next generation sequencing of microRNAs from isogenic neuroblastoma cell lines isolated before and after treatment. Cancer Lett. 372, 128-136 https://doi.org/10.1016/j.canlet.2015.11.026

Rottiers V, Najafi-Shoushtari SH, Kristo F, Gurumurthy S, Zhong L, Li Y, Cohen DE, Gerszten RE, Bardeesy N, Mostoslavsky R, Näär AM (2011): MicroRNAs in metabolism and metabolic diseases. Cold Spring Harbor symposia on quantitative biology $76,225-233$ https://doi.org/10.1101/sqb.2011.76.011049

Stumpf M, den Hertog J (2016): Differential requirement for pten lipid and protein phosphatase activity during zebrafish embryonic development. PLoS One 11, e0148508 https://doi.org/10.1371/journal.pone.0148508

Tate G, Suzuki T, Nemoto H, Kishimoto K, Hibi K, Mitsuya T (2009): Allelic loss of the PTEN gene and mutation of the TP53 gene in choriocarcinoma arising from gastric adenocarcinoma: analysis of loss of heterozygosity in two male patients with extragonadal choriocarcinoma. Cancer Genet. Cytogenet. 193, 104-108 https://doi.org/10.1016/j.cancergencyto.2009.04.005

Tian D, Yang Q, Dong Q, Li N, Yan B, Fan D (2018): Trends in stroke subtypes and vascular risk factors in a stroke center in China over 10 years. Sci. Rep. 8, 5037 https://doi.org/10.1038/s41598-018-23356-9

Tielking K, Fischer S, Preissner KT, Vajkoczy P, Xu R (2019): Extracellular RNA in central nervous system pathologies. Front. Mol. Neurosci. 12, 254 https://doi.org/10.3389/fnmol.2019.00254

Ventura A, Young AG, Winslow MM, Lintault L, Meissner A, Erkeland SJ, Newman J, Bronson RT, Crowley D, Stone JR, et al. (2008): Targeted deletion reveals essential and overlapping functions of the miR-17 through 92 family of miRNA clusters. Cell 132, 875-886 https://doi.org/10.1016/j.cell.2008.02.019

Verklan MT (2009): The chilling details: hypoxic-ischemic encephalopathy. J. Perinat. Neonatal Nurs. 23, 59-68 https://doi.org/10.1097/01.JPN.0000346221.48202.7e

Vinters HV, Zarow C, Borys E, Whitman JD, Tung S, Ellis WG, Zheng L, Chui HC (2018): Review: Vascular dementia: clinicopathologic and genetic considerations. Neuropathol. Appl. Neurobiol. 44, 247-266 https://doi.org/10.1111/nan.12472

Wang J, Ning R, Wang Y (2016): Plasma D-dimer level, the promising prognostic biomarker for the acute cerebral infarction patients. J. Stroke Cerebrovasc. Dis. 25, 2011-2015 https://doi.org/10.1016/j.jstrokecerebrovasdis.2015.12.031

Wei N, Xiao L, Xue R, Zhang D, Zhou J, Ren H, Guo S, Xu J (2016): MicroRNA-9 mediates the cell apoptosis by targeting Bcl2111 in ischemic stroke. Mol. Neurobiol. 53, 6809-6817 https://doi.org/10.1007/s12035-015-9605-4

Zhang X, Chen Y, Zhao P, Zang L, Zhang Z, Wang X (2017): MicroRNA-19a functions as an oncogene by regulating PTEN/AKT/ pAKT pathway in myeloma. Leuk. Lymphoma 58, 932-940 https://doi.org/10.1080/10428194.2016.1213827

Zhao H, Sapolsky RM, Steinberg GK (2006): Phosphoinositide3-kinase/akt survival signal pathways are implicated in neuronal survival after stroke. Mol. Neurobiol. 34, 249-270 https://doi.org/10.1385/MN:34:3:249

Zhu YM, Wang CC, Chen L, Qian LB, Ma LL, Yu J, Zhu MH, Wen CY, Yu LN, Yan M (2013): Both PI3K/Akt and ERK1/2 pathways participate in the protection by dexmedetomidine against transient focal cerebral ischemia/reperfusion injury in rats. Brain Res. 1494, 1-8 https://doi.org/10.1016/j.brainres.2012.11.047

Zou JB, Chai HB, Zhang XF, Guo DY, Tai J, Wang Y, Liang YL, Wang F, Cheng JX, Wang J, Shi YJ (2019): Reconstruction of the lncRNA-miRNA-mRNA network based on competitive endogenous RNA reveal functional lncRNAs in cerebral infarction. Sci. Rep. 9, 12176 https://doi.org/10.1038/s41598-019-48435-3

Received: September 3, 2019

Final version accepted: January 10, 2020 